01

\title{
Электронная структура интерметаллического соединения GdCuGe
}

\author{
(C) А.В. Лукоянов ${ }^{1,2}$, Ю.В. Князев ${ }^{1}$, Ю.И. Кузьмин ${ }^{1}$ \\ ${ }^{1}$ Институт фризики металлов УрО РАН, \\ Екатеринбург, Россия \\ ${ }^{2}$ Уральский федеральный университет им. Б.Н. Ельцина, \\ Екатеринбург, Россия \\ E-mail: lukoyanov@imp.uran.ru
}

(Поступила в Редакцию 2 октября 2017 г.)

\begin{abstract}
Исследована электронная структура интерметаллида GdCuGe. Выполнены спин-поляризованные расчеты энергетического спектра зонными методами с учетом сильных электронных корреляций в 4f-оболочке ионов гадолиния. В теоретическом расчете получено антиферромагнитное упорядочение $\mathrm{GdCuGe}$ при низких температурах, величина эффективного магнитного момента ионов гадолиния воспроизведена в хорошем согласии с экспериментальными данными. Проанализирована структура плотности электронных состояний, рассчитан спектр оптической проводимости $\mathrm{GdCuGe}$, в котором выявлены особенности, аналогичные обнаруженным ранее в соединении GdCuSi co схожей гексагональной структурой.
\end{abstract}

Работа выполнена в рамках государственного задания ФАНО России (тема „Электрон“, № 01201463326) при частичной поддержке РФФИ (проекты 16-52-48012, 17-52-45056). А.В. Лукоянов благодарит за поддержку Правительство Российской Федерации (постановление № 211, контракт № 02.А03.21.0006).

DOI: $10.21883 /$ FTT.2018.04.45667.288

\section{1. Введение}

Тройные интерметаллические соединения эквиатомного состава на основе редкоземельных металлов привлекают повышенное внимание исследователей, что связано с обнаружением в ряде таких материалов гигантского магнетокалорического эффекта (МКЭ), перспективного для применения в устройствах магнитного охлаждения [1]. Самым известным из интерметаллидов такого типа является $\mathrm{Gd}_{5} \mathrm{Si}_{2} \mathrm{Ge}_{2}$, в котором величина МКЭ показывает рекордные значения [2]. В последние годы активно изучаются соединения серии RTX $(\mathrm{R}-$ редкоземельный металл, $\mathrm{T}-d$ - или $p$-металл, $\mathrm{X}$ - p-элемент). В частности, ряд магнитных и электронных свойств исследован в системах $\mathrm{RCuGe}(\mathrm{R}=\mathrm{Ce}$, Pr, Nd, Tb, Dy, Ho, Er) [3-6], а также GdCuSi [7,8] и $\mathrm{GdCuMg}$ [9]. Все перечисленные соединения при низких температурах проявляют магнитное упорядочение ферромагнитного или антиферромагнитного типа. Помимо значительных по величине МКЭ, большие магнитострикционные и магниторезистивные эффекты, обнаруженные в данных интерметаллидах, также представляют интерес для функционального использования. Большое разнообразие физических свойств таких материалов мотивирует исследование их электронной структуры.

В данной работе зонными методами выполнены расчеты энергетического спектра соединения $\mathrm{GdCuGe}$. Нейтронографические исследования, а также измерения намагниченности, теплоемкости и магнитной восприимчивости [10-13] свидетельствуют о наличии в данном сплаве антиферромагнитного упорядочения си- нусоидального типа с температурой Нееля $T_{N}=17 \mathrm{~K}$. Изучение кристаллической структуры интерметаллида проводили в работе [13].

\section{2. Метод расчета}

Соединение GdCuGe кристаллизуется в гексагональной структуре типа $\mathrm{NdPtSb}$, пространственная группа симметрии $\mathrm{P6}_{3}$ mc (номер группы 186). Кристаллическая структура $\mathrm{GdCuGe}$ представлена на рис. 1. Атомы $\mathrm{Cu}$ и $\mathrm{Ge}$ (показаны малыми темными и светлыми шарами) образуют гексагоны в плоскости $a b$, над и под центрами которых располагаются атомы Gd. В расчетах использовались экспе-

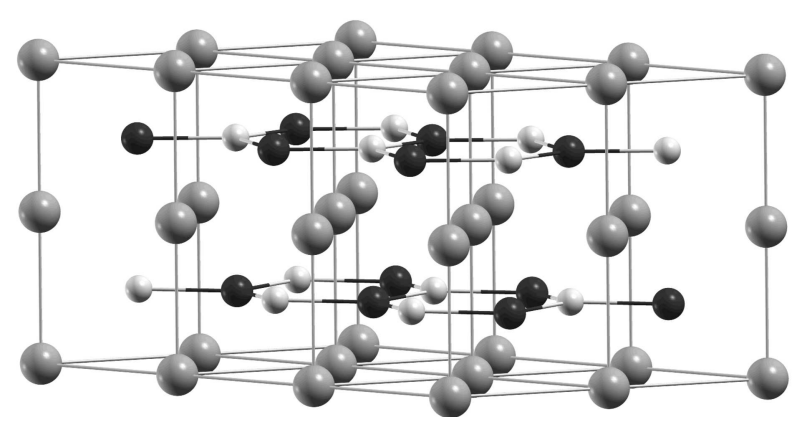

Рис. 1. Кристаллическая структура GdCuGe. Атомы гадолиния представлены большими серыми, меди - малыми темными, германия - малыми светлыми шарами. (При построении данного рисунка использовался программный пакет XCrySDen [14]). 
риментальные величины параметров кристаллической решетки [13]: $a=4.232(1), c=7.537(2) \AA . \quad$ В соединении $\mathrm{GdCuGe}$ ионы $\mathrm{Gd}$ располагаются в позициях типа $2 a(0 ; 0 ; 0), \mathrm{Cu}-2 b(2 / 3 ; 1 / 3 ; 0.7436(5))$, $\mathrm{Ge}-2 b(2 / 3 ; 1 / 3 ; 0.7436(5))[10]$. Элементарная ячейка $\mathrm{GdCuGe}$ в данной структуре содержит две формульные единицы.

Расчеты электронной структуры выполнены методом LSDA + U [15], который в приближении локальной электронной спиновой плотности учитывает поправку на сильные электронные корреляции в $4 f$-оболочке иона $\mathrm{Gd}$. Использовался пакет программ TB-LMTO-ASA, версия 47 [16], метод линеаризованных маффин-тин орбиталей в приближении атомных сфер. Интегрирование в обратном пространстве осуществлялось по сетке k-точек с полным числом точек $10 \times 10 \times 8=800$. В орбитальный базис были включены маффин-тин (МТ) орбитали, соответствующие $6 s-$, $6 p-$, $5 d$ - и $4 f$-состояниям $\mathrm{Gd}, 4 s-, 4 p$ - и $3 d$-состояниям $\mathrm{Cu}$, а также $4 s$-, $4 p$ - и $4 d$-состояниям Ge. Радиус MT сферы Gd составлял 3.8 a.u. и 2.6 a.u. для $\mathrm{Cu}$ и $\mathrm{Ge}$, аналогично значениям для соответствующих ионов в $\mathrm{GdCuSi}[7,8]$.

\section{3. Результаты и обсуждение}

В самосогласованных расчетах электронной структуры $\mathrm{GdCuGe}$ в рамках метода LSDA + U были использованы общепринятые величины параметров прямого кулоновского $U$ и обменного $J$ взаимодействий для $4 f$-оболочки $\mathrm{Gd}: U=6.7 \mathrm{eV}$ и $J=0.7 \mathrm{eV}$ [15]. $\mathrm{B}$ результате проведенных расчетов ионы $\mathrm{Cu}$ и $\mathrm{Ge}$ в $\mathrm{GdCuGe}$ оказались полностью немагнитными, полученные значения магнитных моментов составили $<0.01 \mu_{\mathrm{B}}$. Рассчитанный магнитный спиновый момент ионов $\mathrm{Gd}$ составил $6.90 \mu_{\mathrm{B}}$, что позволяет вычислить для фактора Ланде $g=2$ эффективный момент, равный $7.84 \mu_{\mathrm{B}}$. Полученная величина находится в хорошем согласии с экспериментальным значением $7.89 \mu_{\mathrm{B}}[11]$, полученным из экспериментальных данных по магнитной восприимчивости.

При проведении расчетов электронной структуры исследовались антиферромагнитное (АФМ) и ферромагнитное (ФМ) упорядочения магнитных моментов редкоземельных ионов $\mathrm{Gd}$. Рассчитанные полные энергии составили $E(\Phi \mathrm{M})=-60058.7 \mathrm{Ry}, E(\mathrm{AФM})=$ $=-60058.8 \mathrm{Ry}$, антиферромагнитное решение ниже на $0.1 \mathrm{Ry}(1.4 \mathrm{eV})$ и определяет основное состояние $\mathrm{GdCuGe}$ при низких температурах в согласии с экспериментальными данными $[8,9]$.

На рис. 2, $а$ представлены рассчитанные полные плотности электронных состояний интерметаллического соединения $\mathrm{GdCuGe}$ для двух противоположных направлений спина $\uparrow$ и $\downarrow$. Также приведены парциальные плотности состояний для $4 f$-электронов $\mathrm{Gd}$ и $3 d$-электронов $\mathrm{Cu}(b), 5 d$-электронов $\mathrm{Gd}$ и $4 p$-электронов $\mathrm{Cu}(c)$, $4 s$ - и $4 p$-электронов $\mathrm{Ge}(d)$. Плотности электронных

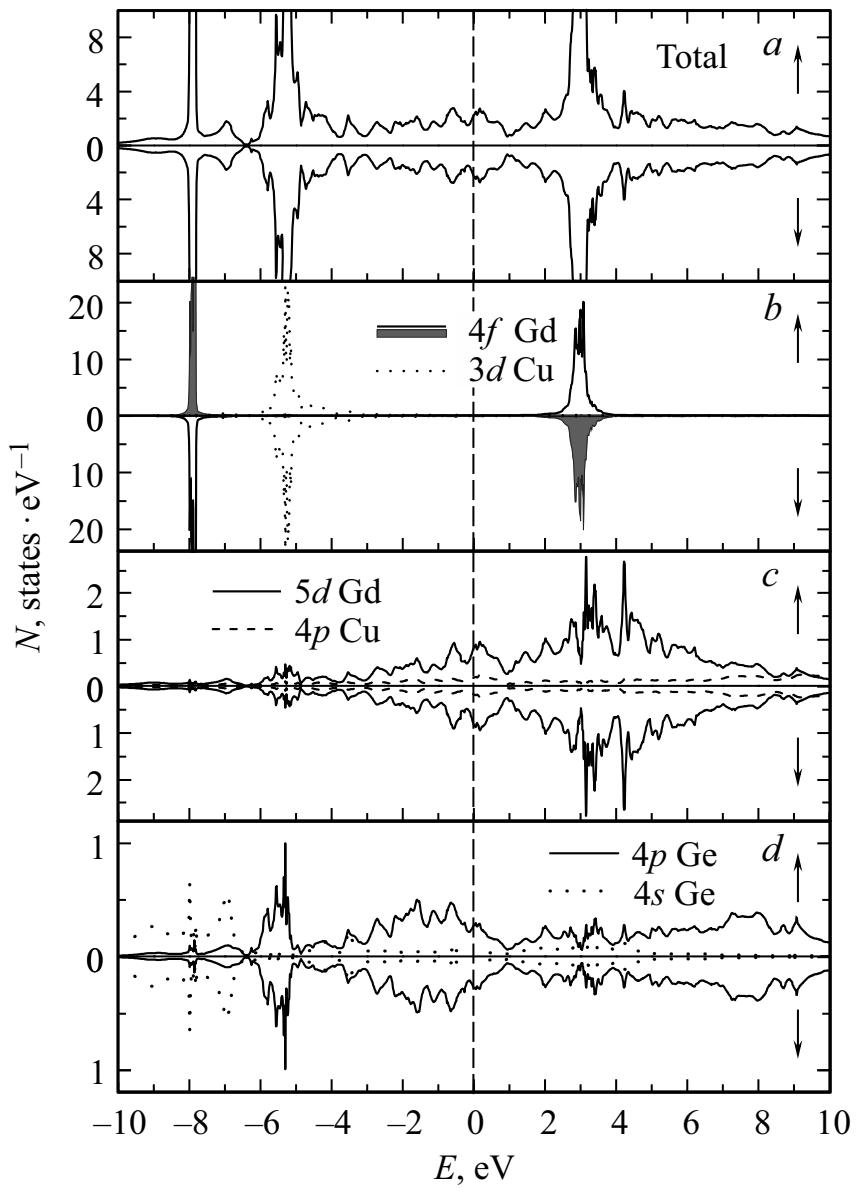

Рис. 2. Полная $(a)$ и парциальные $(b-d)$ плотности электронных состояний соединения $\mathrm{GdCuGe}$. Сплошная линия и затемненные области на панели $b$ соответствуют $4 f$ состояниям $\mathrm{Gd}$ с противоположно направленными спиновыми моментами. Уровень Ферми соответствует нулю на шкале энергий.

состояний с наибольшей интенсивностью достаточно удалены от уровня Ферми $\left(E_{\mathrm{F}}\right)$. Интенсивные и узкие пики полной плотности состояний определяются локализованными $4 f$-состояниями $\mathrm{Gd}$. Эти состояния в заполненной подзоне имеют вид одиночного острого пика, на $8 \mathrm{eV}$ ниже $E_{\mathrm{F}}$. При этом в незаполненной части плотности состояний благодаря учету корреляций $4 f$-электронов данные состояния находятся в интервале $3-4 \mathrm{eV}$ для обоих направлений магнитного момента. Электронные $3 d$-состояния меди (рис. 3,a) образуют полностью заполненную неспинполяризованную зону, расположенную в пределах $3-7 \mathrm{eV}$ ниже $E_{\mathrm{F}}$. Вблизи уровня Ферми полная плотность состояний представлена широкой полосой $5 d$-электронных состояний гадолиния, а электронные состояния $4 p-\mathrm{Cu}(c), 4 p$ - и $4 s-\mathrm{Ge}(d)$ обладают гораздо меньшей интенсивностью и также располагаются в широком энергетическом интервале. Наблюдается слабое смешивание $5 d$ - и $4 f$-состояний $\mathrm{Gd}$ за счет эффектов гибридизации. 


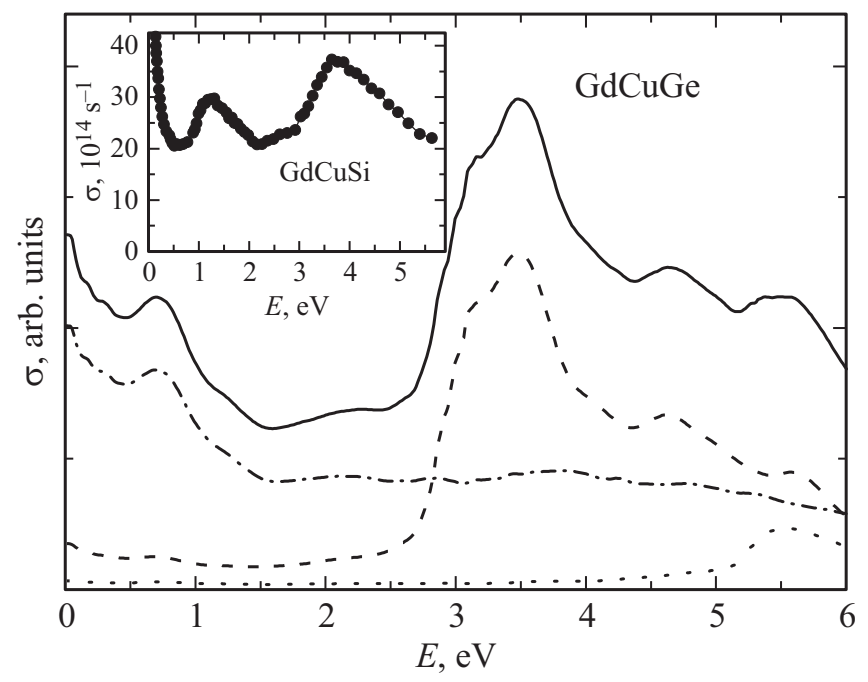

Pис. 3. Рассчитанные спектры межзонной оптической проводимости соединения $\mathrm{GdCuGe}$ (в произвольных единицах). Сплошная кривая - расчет из плотностей состояний с учетом правил отбора по квантовому числу. Штриховая, штрихпунктирная и пунктирная линии - вклады от переходов $4 f-5 d \mathrm{Gd}, 5 d-6 p$ Gd и $3 d-4 p \mathrm{Cu}$ соответственно. На вставке для сравнения приведена экспериментальная кривая оптической проводимости близкого соединения $\mathrm{GdCuSi}$ [7].

Обсуждаемая выше картина дисперсионной зависимости полной плотности электронных состояний $\mathrm{GdCuGe}$ дает возможность рассчитать оптическую проводимость $\sigma(\omega)$ данного соединения, непосредственно связанную c межзонным поглощением света. Для определения межзонного вклада в $\sigma(\omega)$ на базе зонного расчета выполнена свертка парциальных плотностей состояний на каждом неэквивалентном ионе элементарной ячейки с учетом правил отбора по квантовым числам $n^{\prime}=n \pm 1$. Затем проведено суммирование всех полученных результатов в соответствии с количеством ионов данного типа в элементарной ячейке. Оказалось, что наиболее существенный вклад в конечную величину дают лишь свертки по $4 f-5 d$ и $5 d-6 p$ состояниям ионов $\mathrm{Gd}$, тогда как все остальные имеют существенно меньшую величину. При этом свертки $4 f-5 d$ состояний на ионах Gd формируют интенсивную полосу поглощения при $3-4 \mathrm{eV}$, а интегральные величины для $5 d-6 p$ состояний определяют структуру с максимумом вблизи энергии $0.7 \mathrm{eV}$. На рис. 3 представлена сумма всех сверток по ионам элементарной ячейки, а также отдельно показаны свертки по $4 f-5 d$ и $5 d-6 p$ состояниям ионов $\mathrm{Gd}$ и $3 d-4 p$ состояниям ионов $\mathrm{Cu}$, ответственные за образование двух основных особенностей на кривой межзонной оптической проводимости. Характерно, что $5 d-6 p$ переходы на ионах Gd существенны во всем исследуемом энергетическом интервале, тогда как $4 f-5 d$ переходы дают значительный вклад в ограниченной области спектра - при энергиях выше $\sim 2.7 \mathrm{eV}$. Экспериментальные исследования спектральных свойств $\mathrm{GdCuGe}$ до настоя- щего времени не проводились. Поэтому мы сравниваем результаты данного расчета $\sigma(\omega)$ с оптической проводимостью гексагонального соединения $\mathrm{GdCuSi}$ (вставка на рис. 3) - материала, по данным работы [7], имеющего почти идентичную структуру электронного спектра. Сопоставление показывает, что суперпозиция всех сверток довольно хорошо воспроизводит общую форму дисперсионной зависимости оптической проводимости GdCuSi. Расчет качественно отображает главные особенности экспериментального спектра $\sigma(\omega)-$ наличие двух интенсивных максимумов, формируемых различными типами электронных переходов.

\section{4. Заключение}

При помощи самосогласованных спин-поляризованных LSDA + U расчетов исследована электронная структура интерметаллида $\mathrm{GdCuGe}$. Учет сильных электронных корреляций для $4 f$-состояний $\mathrm{Gd}$ позволил корректно описать основное антиферромагнитное состояние $\mathrm{GdCuGe}$ при низких температурах, а также воспроизвести величину эффективного магнитного момента ионов $\mathrm{Gd}$ в хорошем согласии с экспериментальными данными. Полученные в результате расчетов полная и парциальные плотности электронных состояний $\mathrm{GdCuGe}$ позволяют выявить основные особенности электронной структуры соединения, определить состояния, формирующие спектр оптической проводимости при различных энергиях межзонного поглощения. По картине частотной зависимости данной характеристики $\mathrm{GdCuGe}$ оказался близок к изученному ранее интерметаллическому соединению $\mathrm{GdCuSi}$ со схожей гексагональной структурой.

\section{Список литературы}

[1] J. Lyubina. J. Phys. D 50, 053002 (2017).

[2] V.K. Pecharsky, K.A. Gschneidner. Phys. Rev. Lett. 78, 4494 (1997).

[3] F. Yang, J.P. Kuang, J. Li, E. Brück, H. Nakotte, F.R. de Boer, X. Wu, Z. Li, Y. Wang. J. Appl. Phys. 69, 4705 (1991).

[4] S. Gupta, K.G. Suresh. J. Magn. Magn. Mater. 391, 151 (2015).

[5] A.M. Strydom, B.M. Sondezi-Mhlungu. J. Phys.: Conf. Series 200, 032071 (2010).

[6] V. Goruganti, K.D.D. Rathnayaka, J.H. Ross Jr., Y. Öner, C.S. Lue, Y.K. Kuo. J. Appl. Phys. 103, 073919 (2008).

[7] Yu.V. Knyazev, A.V. Lukoyanov, Yu.I. Kuz'min, S. Gupta, K.G. Suresh. Physica B 487, 85 (2016).

[8] S. Gupta, K.G. Suresh, A.V. Lukoyanov. J. Mater. Sci. 50, 5723 (2015).

[9] S. Stein, L. Heletta, R. Pöttgen, Z. Naturforsch. B 72, 511 (2017). 
[10] H. Oesterreicher. Phys. Status Solidi A 39, K75 (1977).

[11] S. Baran, A. Szytułe la J. Leciejewicz, N. Stusser, A. Zygmunt, Z. Tomkowicz, M. Guillot. J. Alloys Compd. 243, 112 (1996).

[12] S. Baran, A. Szytula J. Leciejewicz, N. Stüsser, A. Zygmunt, Z. Tomkowicz, M. Guillot. Physica B 234-236, 656 (1997).

[13] S. Rayaprol, C.P. Sebastian, R. Pöttgen. J. Solid State Chem. 179, 2041 (2006).

[14] A. Kokalj. Comput. Mater. Sci. 28, 155 (2003).

[15] V.I. Anisimov, F. Aryasetiawan, A.I. Lichtenstein. J. Phys.: Condens. Matter 9, 767 (1997).

[16] O.K. Andersen, O. Jepsen. Phys. Rev. Lett. 53, 2571 (1984). 